\title{
Variations spatiotemporelles des compartiments autotrophes et hétérotrophes de la boucle microbienne dans les lacs du sud du Québec
}

\section{Spatial and temporal variations in autotrophic and heterotrophic compartments of the microbial loop in southern Quebec lakes}

\author{
B. Pinel-Alloul, N. Bourbonnais et F. Pick
}

Volume 15, numéro 1, 2002

URI : https://id.erudit.org/iderudit/705432ar

DOI : https://doi.org/10.7202/705432ar

Aller au sommaire du numéro

\section{Éditeur(s)}

Université du Québec - INRS-Eau, Terre et Environnement (INRS-ETE)

\section{ISSN}

0992-7158 (imprimé)

1718-8598 (numérique)

Découvrir la revue

Citer cet article

Pinel-Alloul, B., Bourbonnais, N. \& Pick, F. (2002). Variations spatiotemporelles des compartiments autotrophes et hétérotrophes de la boucle microbienne dans les lacs du sud du Québec. Revue des sciences de l'eau / Journal of Water Science, 15(1), 3-25. https://doi.org/10.7202/705432ar

\section{Résumé de l'article}

Les variations de la biomasse des compartiments autotrophes et hétérotrophes de la boucle microbienne ont été examinées dans neuf lacs de deux régions du sud du Québec. Six lacs étaient situés dans les Laurentides et trois lacs dans les Cantons de l'Est. Ils se différenciaient en fonction de leur statut trophique, de la géologie du bassin versant, et de la physicochimie des eaux. Dans chaque lac, l'échantillonnage a été réalisé à trois profondeurs correspondant aux strates épi-, méta- et hypolimnétiques et à cinq dates au cours de l'été (1990-1992). Les biomasses moyennes de picoplancton autotrophe (PPA : 16-80 $\mu \mathrm{g} \cdot \mathrm{C} \cdot \mathrm{L}^{-1}$ ), de picoplancton hétérotrophe (PPH : 97-647 $\mu \mathrm{g} \cdot \mathrm{C} \cdot \mathrm{L}^{-1}$ ), de nanoplancton autotrophe (NPA : 7-37 $\mu \mathrm{g} \cdot \mathrm{C} \cdot \mathrm{L}^{-1}$ ) et de nanoplancton hétérotrophe (NPH : 9-29 $\mu \mathrm{g} \cdot \mathrm{C} \cdot \mathrm{L}^{-1}$ ) notées dans les lacs du sud du Québec le long d'un gradient trophique de PT variant de 7 à $73 \mu \mathrm{g} \cdot \mathrm{L}^{-1}$ étaient du même ordre de grandeur que celles rapportées pour d'autres écosystèmes d'eau douce au Canada.

La majeure partie de la variation dans les biomasses des compartiments microbiens étaient reliée aux variations inter-lacs mais il existaient aussi des sources de variation significatives au sein des lacs, soit au niveau spatial entre les strates limnétiques ou au niveau temporel entre les dates d'échantillonnage durant l'été. Toutefois, les patrons de variation spatiotemporelle intra-lac variaient d'un lac à l'autre. Les variations inter-lacs de la biomasse des quatre compartiments microbiens ont été mises en relation avec les changements dans les concentrations de phosphore total (PT) et dans les densités de macrozooplancton. Le niveau trophique des lacs, exprimés en PT, et l'abondance du macrozooplancton avaient un effet sur les biomasses des compartiments picoplanctoniques. La biomasse de PPA et PPH décroissait dans les lacs eutrophes des Cantons de l'Est ayant des concentrations de PT supérieures à $20 \mu \mathrm{g} \cdot \mathrm{L}^{-1}$ et dans les lacs ayant de fortes densités de macrozooplancton ou un faible rapport micro-macrozooplancton. Notre étude n'a pas mis clairement en évidence les effets du niveau trophique ou du macrozoopancton sur les compartiments nanoplanctoniques (NPA, NPH).
Ce document est protégé par la loi sur le droit d'auteur. L’utilisation des services d'Érudit (y compris la reproduction) est assujettie à sa politique d'utilisation que vous pouvez consulter en ligne.

https://apropos.erudit.org/fr/usagers/politique-dutilisation/ 


\title{
Variations spatiotemporelles des compartiments autotrophes et hétérotrophes de la boucle microbienne dans les lacs du sud du Québec
}

\author{
Spatial and temporal variations in autotrophic \\ and heterotrophic compartments of the microbial \\ loop in southern Quebec lakes
}

\author{
B. PINEL-ALLOUL ${ }^{1}{ }^{*}$, N. BOURBONNAIS ${ }^{2}$, F. $^{\text {PICK }}{ }^{3}$
}

\section{SUMMARY}

The biomass of autotrophic and heterotrophic microbial compartments were measured in nine lakes in two regions of southern Quebec. Six lakes were located in the Laurentides while three lakes were situated in the Eastern Townships. They varied in trophic status, watershed geology, and water chemistry. Each lake was sampled at three depths, corresponding to the epi-, meta-, and hypolimnion strata, and at five dates over the summer season (1991-1992). The mean biomass of autotrophic picoplankton (PPA: 16$80 \mu \mathrm{g} \cdot \mathrm{C} \cdot \mathrm{L}^{-1}$ ), heterotrophic picoplankton (PPH: 97-647 $\mu \mathrm{g} \cdot \mathrm{C} \cdot \mathrm{L}^{-1}$ ), autotrophic nanoplankton (NPA: $7-37 \mu \mathrm{g} \cdot \mathrm{C} \cdot \mathrm{L}^{-1}$ ), and heterotrophic nanoplankton (NPH: 9-29 $\mu \mathrm{g} \cdot \mathrm{C} \cdot \mathrm{L}^{-1}$ ) found in Quebec lakes over a PT range of trophy varying from 7 to $73 \mu \mathrm{g} \cdot \mathrm{L}^{-1}$ were of the same order as those reported in other freshwater environments in Canada.

Most of the variation in the biomass of the four microbial compartments was related to among-lake variation. Some variation was also due to within-lake spatial variation among limnetic strata or temporal variation over the summer season. However, within-lake patterns of variation patterns were different among lakes. Among-lake variations in the biomass of the four microbial compartments were examined in relation to changes in total phosphorus concentrations (PT) and macrozooplankton densities between lakes. Both lake trophy, expressed by PT, and macrozooplankton abundance influence the biomass of picoplankton compartments. The biomass of PPA and PPH decline in the most eutrophic lakes of the Eastern Townships where PT $>20 \mu \mathrm{g} \cdot \mathrm{L}^{-1}$. PPA and PPH biomass were also reduced in lakes with the

1. GRIL, Département de sciences biologiques, Université de Montréal, CP 6128, succ. Centre ville. Montréal, Qué. H3C 3J7. Canada.

2. Ministère de l'environnement et de la Faune, Gouvernement du Québec, Sept-Îles, Qué. H3C 3 P8. Canada.

3. Département de biologie, Université d'Ottawa, Ottawa, Ontario, K1N 6N5, Canada.

* Correspondance. E-mail : bernadette.pinel-alloul@umontreal.ca

Les commentaires seront reçus jusqu'au 31 mars 2003. 
highest density of macrozooplankton or with low micro-/macrozooplankton ratio. Our study did not clearly detected the effects of lake trophy or macrozooplankton on nanoplanktonic compartments.

Key-words: picoplankton, nanoplankton, microbial loop, lakes, southern Quebec.

\section{RÉSUMÉ}

Les variations de la biomasse des compartiments autotrophes et hétérotrophes de la boucle microbienne ont été examinées dans neuf lacs de deux régions du sud du Québec. Six lacs étaient situés dans les Laurentides et trois lacs dans les Cantons de l'Est. Ils se différenciaient en fonction de leur statut trophique, de la géologie du bassin versant, et de la physicochimie des eaux. Dans chaque lac, l'échantillonnage a été réalisé à trois profondeurs correspondant aux strates épi-, méta- et hypolimnétiques et à cinq dates au cours de l'été (1990-1992). Les biomasses moyennes de picoplancton autotrophe (PPA : 16-80 $\left.\mu \mathrm{g} \cdot \mathrm{C} \cdot \mathrm{L}^{-1}\right)$, de picoplancton hétérotrophe (PPH : 97 $\left.647 \mu \mathrm{g} \cdot \mathrm{C} \cdot \mathrm{L}^{-1}\right)$, de nanoplancton autotrophe $\left(\mathrm{NPA}: 7-37 \mu \mathrm{g} \cdot \mathrm{C} \cdot \mathrm{L}^{-1}\right.$ ) et de nanoplancton hétérotrophe (NPH : 9-29 $\mu \mathrm{g} \cdot \mathrm{C} \cdot \mathrm{L}^{-1}$ ) notées dans les lacs du sud du Québec le long d'un gradient trophique de PT variant de 7 à $73 \mu \mathrm{g} \cdot \mathrm{L}^{-1}$ étaient du même ordre de grandeur que celles rapportées pour d'autres écosystèmes d'eau douce au Canada.

La majeure partie de la variation dans les biomasses des compartiments microbiens étaient reliée aux variations inter-lacs mais il existaient aussi des sources de variation significatives au sein des lacs, soit au niveau spatial entre les strates limnétiques ou au niveau temporel entre les dates d'échantillonnage durant l'été. Toutefois, les patrons de variation spatiotemporelle intralac variaient d'un lac à l'autre. Les variations inter-lacs de la biomasse des quatre compartiments microbiens ont été mises en relation avec les changements dans les concentrations de phosphore total (PT) et dans les densités de macrozooplancton. Le niveau trophique des lacs, exprimés en PT, et l'abondance du macrozooplancton avaient un effet sur les biomasses des compartiments picoplanctoniques. La biomasse de PPA et PPH décroissait dans les lacs eutrophes des Cantons de l'Est ayant des concentrations de PT supérieures à $20 \mu \mathrm{g} \cdot \mathrm{L}^{-1}$ et dans les lacs ayant de fortes densités de macrozooplancton ou un faible rapport micro-macrozooplancton. Notre étude n'a pas mis clairement en évidence les effets du niveau trophique ou du macrozoopancton sur les compartiments nanoplanctoniques (NPA, NPH).

Mots clés : picoplancton, nanoplancton, boucle microbienne, lacs, sud du Québec.

\section{1 - INTRODUCTION}

Depuis quelques décennies, de nombreuses études ont tenté de documenter et de comprendre le rôle écologique de la boucle microbienne dans les réseaux planctoniques des écosystèmes lacustres (CARON et al., 1988 ; STOCKNER et PORTER, 1988 ; CHRISTOFFERSEN et al., 1993 ; AMBLARD et al., 1996). II est maintenant reconnu que les compartiments autotrophes et hétérotrophes du picoplancton et du nanoplancton contribuent significativement à la biomasse et la productivité biologique des lacs et qu'ils jouent un rôle prépondérant dans le transfert de carbone vers les niveaux trophiques supérieurs 
(RIEMANN et SONDERGAARD, 1986 ; PICK et CARON, 1987 ; STOCKNER et SHORTREED, 1989 ; WEISSE, 1990 ; MAZUMDER et al., 1990 ; CARRICK et al., 1991 ; NAKANO et al., 1998).

Les variations spatiotemporelles de la biomasse des différents compartiments de la boucle microbienne peuvent être expliquées par les effets de divers facteurs physiques tels que la lumière (PICK, 1991 ; FAHNENSTIEL et CARRICK, 1992), chimiques tels que l'enrichissement en phosphore, azote et carbone organique (WATSON et KALFF, 1981 ; BIRD et KALFF, 1984 ; TRANVIK, 1992 ; WEHR 1993 ; NÜRNBERG et SHAW, 1998 ; TZARAS et al., 1999 ; JANSSON et al., 1999) ou biologiques tels que la production ou la biomasse des algues (LETARTE et al., 1992 ; PINEL-ALLOUL et LETARTE 1993) et la prédation par le micro- et macrozooplancton (PORTER, 1988 ; NAGATA, 1988 ; SANDERS et al., 1989 ; MAZUMDER et al., 1990 ; CARRICK et al., 1991 ; VAQUÉ et PACE, 1992 ; JÜRGENS, 1994 ; BURNS et SCHALLENBERG, 1998 ; THOUVENOT et al., 1999a).

Toutefois, la plupart des études sur les communautés microbiennes ne traite que d'un seul compartiment à la fois (NAGATA, 1986 ; LETARTE et PINELALLOUL, 1991 ; FAHNENSTIEL et CARRICK, 1992 ; BERTRAND et VINCENT, 1994). Très peu d'études ont évalué simultanément les variations de la biomasse des quatre compartiments de la boucle microbienne : le picoplancton autotrophe (PPA) et hétérotrophe (PPH), le nanoplancton autotrophe (NPA) et hétérotrophe (NPH). Les quelques études portant sur deux (MALINSKY-RUSHANSKY et BERMAN, 1991 ; VAQUÉ et PACE, 1992 ; PINEL-ALLOUL et al., 1996), trois (WEISSE, 1990, 1991) ou même quatre compartiments (PICK et CARON, 1987 ; AMBLARD et al., 1994 ; BERGERON et VINCENT, 1997) n'ont souvent été réalisées que sur un seul lac ou un très petit nombre de lacs.

Dans la présente étude, nous évaluons pour la première fois les variations spatiotemporelles de l'ensemble des compartiments autotrophes (PPA, NPA) et hétérotrophes (PPH, NPH) du réseau microbien dans une série de neuf lacs du Québec de statut trophique et de géomorphologie différents. Cette étude fait suite et complète une recherche précédente portant sur les compartiments autotrophes (PPA et NPA) de six lacs du Québec (PINEL-ALLOUL et al., 1996). L'objectif est de déterminer les variations naturelles inter-lacs et intra-lac (selon la profondeur et durant la saison estivale) de la biomasse des compartiments microbiens et de relier les variations inter-lacs aux changements dans les nutriments et la lumière d'une part, et dans la densité du micro- et macrozooplancton d'autre part.

\section{2 - MATÉRIEL ET MÉTHODES}

\subsection{Sites d'étude (tableau 1)}

Les neuf lacs se répartissent dans deux régions du sud du Québec. Les six lacs de la région des Laurentides (Achigan, Écho, Pin Rouge, Croche, Cromwell et Geai) se situent sur la roche ignée du Bouclier Canadien au nord de Montréal $\left(46^{\circ} \mathrm{N}, 74^{\circ} \mathrm{O}\right)$. Les trois lacs de la région des Cantons de l'Est (Brompton, Magog et Massawippi) sont localisés dans une zone sédimentaire calcaire 
Tableau 1 Caractéristiques morphométriques et moyennes saisonnières des variables physicochimiques pour chaque lac dans les trois sous-ensembles de lacs des Laurentides (1990 et 1992) et des Cantons de l'Est (1991).

Table 1 Morphometric characteristics and seasonal means of physico-chemical variables in each lake of the three sets of lakes in the Laurentides (1990 and 1992) and the Eastern Townships (1991).

\begin{tabular}{|c|c|c|c|c|c|c|c|c|c|c|c|c|c|c|}
\hline Lacs & $\underset{\text { moy. }}{Z}$ & $\underset{\text { (m) }}{Z}$ & $\begin{array}{l}\text { Aire } \\
\left(\mathbf{k m}^{2}\right)\end{array}$ & $\mathrm{pH}$ & $\begin{array}{c}\text { Cond. } \\
\left(\mu \mathrm{S} \cdot \mathrm{cm}^{-1}\right)\end{array}$ & $\begin{array}{c}\text { PT } \\
\left(\mu \mathrm{g} \cdot \mathrm{L}^{-1}\right)\end{array}$ & $\begin{array}{c}\text { PTD } \\
\left(\mu \mathrm{g} \cdot \mathrm{L}^{-1}\right)\end{array}$ & $\begin{array}{c}\mathrm{NO}_{3} \\
\left(\mu \mathrm{g} \cdot \mathrm{L}^{-1}\right)\end{array}$ & $\begin{array}{c}\mathrm{NH}_{4} \\
\left(\mu \mathrm{g} \cdot \mathrm{L}^{-1}\right)\end{array}$ & $\begin{array}{c}\text { Rapport } \\
\text { TN/TP }\end{array}$ & $\begin{array}{c}\text { COD } \\
\left(\mathrm{mg} \cdot \mathrm{L}^{-1}\right)\end{array}$ & $\begin{array}{l}\text { Secchi } \\
\text { (m) }\end{array}$ & $\begin{array}{l}\text { Chlor. } \\
\text { Totale } \\
\left(\mu g \cdot L^{-1}\right)\end{array}$ & $\begin{array}{l}\text { Chlor. } \\
<25 \mu \mathrm{m} \\
\left(\mu \mathrm{g} \cdot \mathrm{L}^{-1}\right)\end{array}$ \\
\hline Achigan & 12,3 & 26,3 & 5,2 & 7,5 & 40,6 & 8,6 & 1,1 & 105,8 & 7,1 & $13: 1$ & 10,5 & 4,5 & 1,9 & 1,6 \\
\hline Écho & 5,9 & 9,1 & 1,8 & 7,9 & 98,0 & 9,6 & 2,5 & 8,8 & 10,4 & $2: 1$ & 16,0 & 3,4 & 3,3 & 3,0 \\
\hline Pin rouge & 8,7 & 14,0 & 0,1 & 6,4 & 48,9 & 7,2 & 2,2 & 118,5 & 43,0 & $22: 1$ & 12,5 & 2,3 & 3,0 & 2,7 \\
\hline $\begin{array}{l}\text { Laurentides } \\
1990\end{array}$ & 9,0 & 16,5 & 2,4 & 7,6 & 62,5 & 8,5 & 1,9 & 77,7 & 20,2 & $11,5: 1$ & 13,0 & 3,4 & 2,7 & 2,4 \\
\hline Brompton & 12,5 & 42,3 & 11,9 & 6,6 & 92,1 & 32,3 & 8,5 & 188,1 & 31,7 & $7: 1$ & 18,2 & 4,0 & 3,5 & 2,7 \\
\hline Magog & 9,8 & 19,2 & 10,8 & 7,0 & 205,5 & 73,0 & 20,4 & 52,8 & 129,2 & $2,5: 1$ & 12,6 & 3,8 & 5,1 & 3,4 \\
\hline Massawippi & 40,2 & 84,8 & 17,9 & 7,5 & 284,7 & 15,8 & 5,4 & 566,6 & 20,3 & $37: 1$ & 12,2 & 4,5 & 4,5 & 3,1 \\
\hline $\begin{array}{l}\text { Cantons } \\
\text { de l'Est } \\
1991\end{array}$ & 20,8 & 48,8 & 13,5 & 7,4 & 194,1 & 40,3 & 11,6 & 260,1 & 60,3 & $8: 1$ & 14,4 & 4,1 & 4,3 & 3,1 \\
\hline Croche & 8,5 & 10,5 & 0,05 & 6,7 & 48,4 & 11,2 & 7,2 & 48,9 & 128,0 & $16: 1$ & 5,9 & 4,8 & 1,9 & 1,3 \\
\hline Cromwell & 3,0 & 9,0 & 0,09 & 5,9 & 45,6 & 14,8 & 9,8 & 25,3 & 125,9 & $10: 1$ & 7,9 & 2,2 & 6,7 & 5,2 \\
\hline Geai & 2,0 & 7,5 & 0,009 & 5,5 & 35,9 & 14,8 & 9,9 & 16,3 & 313,6 & $22: 1$ & 12,5 & 2,4 & 7,6 & 9,3 \\
\hline $\begin{array}{l}\text { Laurentides } \\
1992\end{array}$ & 4,5 & 9,0 & 0,05 & 6,1 & 43,3 & 13,6 & 8,9 & 30,2 & 189,2 & $16: 1$ & 7,9 & 3,1 & 5,4 & 5,3 \\
\hline
\end{tabular}


au sud de Montréal $\left(45^{\circ} \mathrm{N}, 72^{\circ} \mathrm{O}\right)$ (tableau 1). Une description détaillée de la géomorphologie et du couvert forestier des deux régions a été présentée par PINEL-ALLOUL et al. (1996). Le tableau 1 résume les principales caractéristiques morphologiques et trophiques des lacs étudiés de 1990 à 1992. Les lacs des Cantons de l'Est sont en moyenne plus grands (surf. : $13,5 \mathrm{~km}^{2}$ ) et plus profonds (prof. max. : $49 \mathrm{~m}$; prof. moy. : $21 \mathrm{~m}$ ) que les lacs des Laurentides (surf. : 0,05-2,4 km² ; prof. max. : 9-16,5 m ; prof. moy. : 4,5-9 m). Tous les lacs présentaient une bonne stratification avec des températures de l'eau variant de 16 à $24{ }^{\circ} \mathrm{C}$ dans l'épilimnion et de 4 à $10^{\circ} \mathrm{C}$ dans l'hypolimnion. Les lacs différaient au niveau de la qualité chimique des eaux et de leur statut trophique. Dans la région des Laurentides, les lacs avaient des eaux neutres ou légèrement acides ( $\mathrm{pH} 6,1-7,6)$, et peu minéralisées (cond. : 43-62 $\mu \mathrm{S} \cdot \mathrm{cm}^{-1}$ ). Leur niveau trophique variait de oligotrophe à mésotrophe. Les concentrations moyennes de phosphore total (PT : 8,5 à $13,6 \mu \mathrm{g} \cdot \mathrm{L}^{-1}$ ) et de phosphore total dissous (PTD : 1,9 à $8,9 \mu \mathrm{g} \cdot \mathrm{L}^{-1}$ ) étaient plus faibles dans les lacs des Laurentides que dans les lacs des Cantons de l'est (PT : 40,3 $\mu \mathrm{g} \cdot \mathrm{L}^{-1}$; PTD : 11,6 $\mu \mathrm{g} \cdot \mathrm{L}^{-1}$ ). Les concentrations de nitrates $\left(\mathrm{NO}_{3}\right)$ et d'ammoniaque $\left(\mathrm{NH}_{4}\right)$ variaient beaucoup plus d'un lac à l'autre qu'entre les sous-ensembles de lacs. Les plus fortes concentrations de $\mathrm{NH}_{4}$ ont été notées dans les lacs qui présentaient de fortes anoxies dans I'hypolimnion (Geai, Magog, Croche, Cromwell). Les concentrations de carbone organique dissous (COD) dans les lacs des Laurentides ont varié en moyenne de 7,9 à $13 \mathrm{mg} \cdot \mathrm{L}^{-1}$ et la transparence au disque de Secchi de 3,1 à $3,4 \mathrm{~m}$. Dans les lacs des Cantons de l'Est, les concentrations de COD (en moyenne $14,4 \mathrm{mg} \cdot \mathrm{L}^{-1}$ ) étaient légèrement supérieures à celles retrouvées dans les lacs des Laurentides. Toutefois, bien que les concentrations de PT, PTD et $\mathrm{NO}_{3}$ soient plus élevées dans les lacs des Cantons de l'Est, la concentration moyenne de Chlorophylle a (Chlor. a : $4,3 \mu \mathrm{g} \cdot \mathrm{L}^{-1}$ ) n'était pas plus forte et la transparence (Secchi : $4,1 \mathrm{~m}$ ) n'était pas plus faible dans les lacs des Cantons de l'Est que dans ceux des Laurentides. Ceci peut s'expliquer par la forte conductivité des eaux (cond. : $194 \mu \mathrm{S} \cdot \mathrm{cm}^{-1}$ ) dans les lacs des Cantons de l'Est qui les rend sujets à des phénomènes de précipitation calcique et de blanchiment des eaux, entraînant une coprécipitation des algues (ZHANG et PREPAS, 1996) et une plus grande clarté des eaux, en particulier au lac Massawippi.

\section{2 Échantillonnage}

Dans chaque lac, l'échantillonnage a été réalisé à cinq périodes de juin à août dans chacune des strates de l'épilimnion, du métalimnion et de l'hypolimnion définies à partir des profils verticaux d'oxygène et de température. Dans chaque strate, trois échantillons d'eau furent prélevés avec un hydrocapteur Van Dorn (6 litres). Le pH et la conductivité ont été mesurés dans chacune des strates. Un sous-échantillon de $150 \mathrm{~mL}$ d'eau a servi à l'analyse des nutriments (PT, PTD, $\mathrm{NO}_{3}, \mathrm{NH}_{4}, \mathrm{COD}$ ) et 1 litre d'eau a été utilisé pour l'analyse de la chlorophylle a. Pour l'analyse du picoplancton autotrophe et du nanoplancton autotrophe et hétérotrophe, $250 \mathrm{~mL}$ d'eau a été prélevé puis préservé avec du glutaraldéhyde tamponné avec $0,1 \mathrm{M}$ de $\mathrm{Na}$-cacodylate à $\mathrm{pH} 7,2$ pour une concentration finale de $1 \% \mathrm{v} / \mathrm{v}$ (CARON et al., 1985 ; PICK et CARON, 1987). Pour l'analyse du bactérioplancton, $20 \mathrm{~mL}$ d'eau a été récolté dans des fioles stérilisées puis préservé avec du formaldéhyde $2 \%$. Le zooplancton a été récolté dans chaque strate avec une trappe Patalas de 32,5 litres munie d'un 
filtre collecteur de $53 \mu \mathrm{m}$. Les échantillons concentrés de zooplancton $(250 \mathrm{~mL})$ ont été préservés avec du formaldéhyde $2 \%$.

\subsection{Analyses en laboratoire}

Les échantillons d'eau pour l'analyse du PTD et COD ont été filtrés à travers des filtres Whatman GF/F de $0,45 \mu \mathrm{m}$ préalablement brûlés à $500{ }^{\circ} \mathrm{C}$. Les filtrats ont été transférés dans des tubes de $2 \mathrm{~mL}$, préalablement lavés à l'acide nitrique $(15 \% \mathrm{v} / \mathrm{v})$ et rincés à l'eau déionisée, puis congelés à $-20{ }^{\circ} \mathrm{C}$. Les concentrations de PT et de PTD ont été évaluées avec la méthode au bleu de molybdène après digestion au persulfate. Les concentrations de $\mathrm{NO}_{3}$ et $\mathrm{NH}_{4}$ ont été déterminées par conversion des nitrates en nitrites avec la méthode de réduction par le cadmium. Les concentrations de Chlor. a furent estimées pour 2 fractions du phytoplancton : la fraction totale et la fraction $<25 \mu \mathrm{m}$, cette dernière représentant grossièrement les communautés autotrophes du pico$(0,2-2 \mu \mathrm{m})$ et $\mathrm{du}$ nanoplancton $(2-20 \mu \mathrm{m})$. Les lectures ont été faites après extraction avec de l'acétone à $90 \%$ à l'obscurité pendant $24 \mathrm{~h}$; les concentrations de Chlor. $a$ ont été estimées selon la méthode de STRICKLAND et PARSONS (1972), après correction pour les phéopigments selon la méthode d'acidification de LORENZEN (1966).

L'énumération du pico- et du nanoplancton autotrophe et hétérotrophe a été effectuée avec un microscope à épifluorescence Leitz Dialux 20 doté d'une lampe au mercure $\mathrm{HBO}$ de $50 \mathrm{~W}$ et d'une combinaison de filtres pour lumière bleue (filtre excitant BP450-490, filtre barrière LP520, diviseur de faisceau chromatique FT510) et lumière verte (filtre excitant BP510-560, filtre barrière LP590, diviseur de faisceau chromatique FT580) (CARON et al., 1985 ; PICK et CARON, 1987). Les lames ont été préparées en filtrant à faible pression (< $100 \mathrm{~mm} \mathrm{Hg}$ ) (HOBBIE et al., 1977 ; BOOTH, 1993), des sous-échantillons, à travers des filtres Nuclepore $^{\mathrm{TM}}$ en polycarbonate de $0,2 \mu \mathrm{m}$, préalablement colorés au noir d'Irgalan. Un filtre Membrafil de $0,45 \mu \mathrm{m}$ a été installé entre le filtre Nuclepore ${ }^{T M}$ de $0,2 \mu \mathrm{m}$ et la colonne de filtration afin d'assurer la répartition aléatoire des cellules sur les filtres. Pour l'analyse du pico- et du nanoplancton autotrophe, des sous-échantillons de 5 à $20 \mathrm{~mL}$ ont été filtrés. Pour l'analyse du bactérioplancton, les sous-échantillons de 1 à $5 \mathrm{~mL}$ ont été colorés avec du DAPI à une concentration finale de $0,1 \mathrm{mg} \cdot \mathrm{L}^{-1}$ (PORTER et FEIG, 1980) et pour l'analyse du nanoplancton hétérotrophe, les sous-échantillons de 5 à $20 \mathrm{~mL}$ ont été colorés avec de la primuline (CARON, 1983). Les filtres ont par la suite été montés entre lames et lamelles avec de l'huile à immersion Cargille FF, puis immédiatement conservés à $4{ }^{\circ} \mathrm{C}$ afin de minimiser la perte de fluorescence. Les filtres colorés à la primuline ont été conservés à $-20^{\circ} \mathrm{C}$. Les dénombrements microscopiques ont été effectués à un grossissement de $1000 \mathrm{X}$ sur un total de 10 à 15 champs par filtre. Les décomptes de picoplancton autotrophe ont en revanche été poursuivis jusqu'à ce que 400 cellules par filtre soient comptées, afin d'obtenir une précision de $\pm 10 \%$ (LUND et al., 1958). Les cellules de picoet de nanoplancton autotrophe ont été différenciées par leur taille et par leur fluorescence caractéristique. Seules les cellules autotrophes $<20 \mu \mathrm{m}$ furent dénombrées. Sous une excitation lumineuse verte, les picocyanobactéries fluorescaient rouge (PICK, 1991 ; MACISAAC et STOCKNER, 1993). Sous excitation bleue, les picocyanobactéries ayant le pigment phycoérytrhine fluorescaient jaune ou orange tandis que les eucaryotes présentaient une fluorescence 
rouge. Les décomptes de bactérioplancton ont été réalisés sous excitation ultraviolet (UV) tandis que les dénombrements des cellules du nanoplancton hétérotrophe ont été réalisés sous excitation bleue. Les cellules du picoplancton $(0,2-2 \mu \mathrm{m})$ autotrophe et du nanoplancton $(2-20 \mu \mathrm{m})$ autotrophe et hétérotrophe ont été mesurées avec un oculaire micrométrique et le biovolume de chaque cellule a été calculé à partir de formes géométriques simples (VOLLENWEIDER et al., 1974). Le biovolume de chaque type cellulaire fut ensuite calculé en multipliant la densité des différents morphotypes par le biovolume de chaque cellule et la somme de ces biovolumes a permis d'évaluer le biovolume total du pico- et du nanoplancton autotrophe et hétérotrophe. La conversion de ce biovolume en biomasse fut faite en assumant une gravité spécifique de 1 . Les biomasses des différents compartiments déterminées à chaque date et profondeur dans chacun des lacs ont été exprimées en $\mathrm{mg} \cdot \mathrm{m}^{-3}$. Toutefois, afin de permettre les comparaisons entre nos résultats et ceux d'autres études, elles ont ensuite été converties en biomasse de carbone en utilisant comme facteur de conversion $0,22 \mathrm{pg} \cdot \mathrm{C} \cdot \mu \mathrm{m}^{-3}$ pour le picoplancton (BOOTH, 1993) et $0,15 \mathrm{pg} \cdot \mathrm{C} \cdot \mathrm{\mu m}^{-3}$ pour le nanoplancton (CARRICK et FAHNENSTIEL, 1989).

L'identification et le comptage des organismes du zooplancton ont été effectués sur un sous-échantillon de $10 \mathrm{~mL}$ avec une cellule rotative placée sous un binoculaire aux grossissements de 25 ou 50X. La densité des organismes $\left(\mathrm{Nb} \cdot \mathrm{L}^{-1}\right)$ a été calculée en fonction du pourcentage relatif de l'échantillon analysé et du volume d'eau du lac filtrée (PINEL-ALLOUL et al., 1990). Deux classes de taille de zooplancton ont été définies : le microzooplancton composé d'organismes' de taille $<800 \mu \mathrm{m}$ et le macrozooplancton constitué d'organismes de taille $>800 \mu \mathrm{m}$.

\subsection{Analyses statistiques}

Les trois sous-ensembles de lacs (Laurentides 1990, Cantons de l'Est 1991 et Laurentides 1992 : tableau 1) ont été analysés séparément. Les variations spatiales et temporelles de la biomasse du pico- et du nanoplancton autotrophe et hétérotrophe, au sein des lacs d'un même sous-ensemble et entre les différents sous-ensembles, ont été statistiquement évaluées avec un modèle ANOVA modifié (DUTILLEUL, 1993 ; DUTILLEUL et PINEL-ALLOUL, 1996) de façon à tenir compte de l'autocorrélation et l'hétéroscédasticité dans les données. Le facteur lac $(L)$ (facteur = critère de classification) représente la variation spatiale inter-lacs et les facteurs profondeur ( $p: 3$ strates) et date $(d=5$ dates d'échantillonnage) correspondent aux variations spatiotemporelles intra-lac. Ces trois facteurs sont croisés, i.e., tous les lacs ont été échantillonnés à la même date et à la même profondeur (épi-, méta- et hypolimnion). Par conséquent, il existe trois interactions simples (deux facteurs) et un interaction double (trois facteurs) dans le modèle, en plus des effets principaux de chaque facteur ( $L, p$ et $d)$. Le facteur lac $(L)$ a été considéré comme étant aléatoire, i.e., les lacs étudiés sont considérés comme étant un échantillon collecté aléatoirement à partir d'une population de lacs, tandis que les facteurs profondeurs et dates d'échantillonnage ont été choisis en fonction du milieu et de l'expérimentateur. Les trois réplicats d'échantillonnage ont été considérés comme réplicats pour une date et une profondeur donnée dans un lac.

Les relations linéaires entre les biomasses des compartiments autotrophes et hétérotrophes et les variables environnementales (PT, micro- et macrozoo- 
plancton) ont été évaluées statistiquement par un test de t modifié basé sur la statistique $r$ de Pearson (DUTILLEUL, 1993 ; DUTILLEUL et PINEL-ALLOUL, 1996). Afin de respecter la condition de normalité sous-jacente aux tests ANOVA et aux analyses de corrélation, la transformation de Box-Cox-Bartlett (normalisation et stabilisation des variances) (SOKAL et ROHLF, 1981) a été appliquée auX données en utilisant la procédure VERNOM du progiciel R (LEGENDRE et VAUDOR, 1991).

\section{3 - RÉSULTATS ET DISCUSSION}

\subsection{Variations inter-lacs des compartiments microbiens}

La figure 1 présente la variation inter-lacs des biomasses moyennes estivales de chacun des compartiments microbiens.
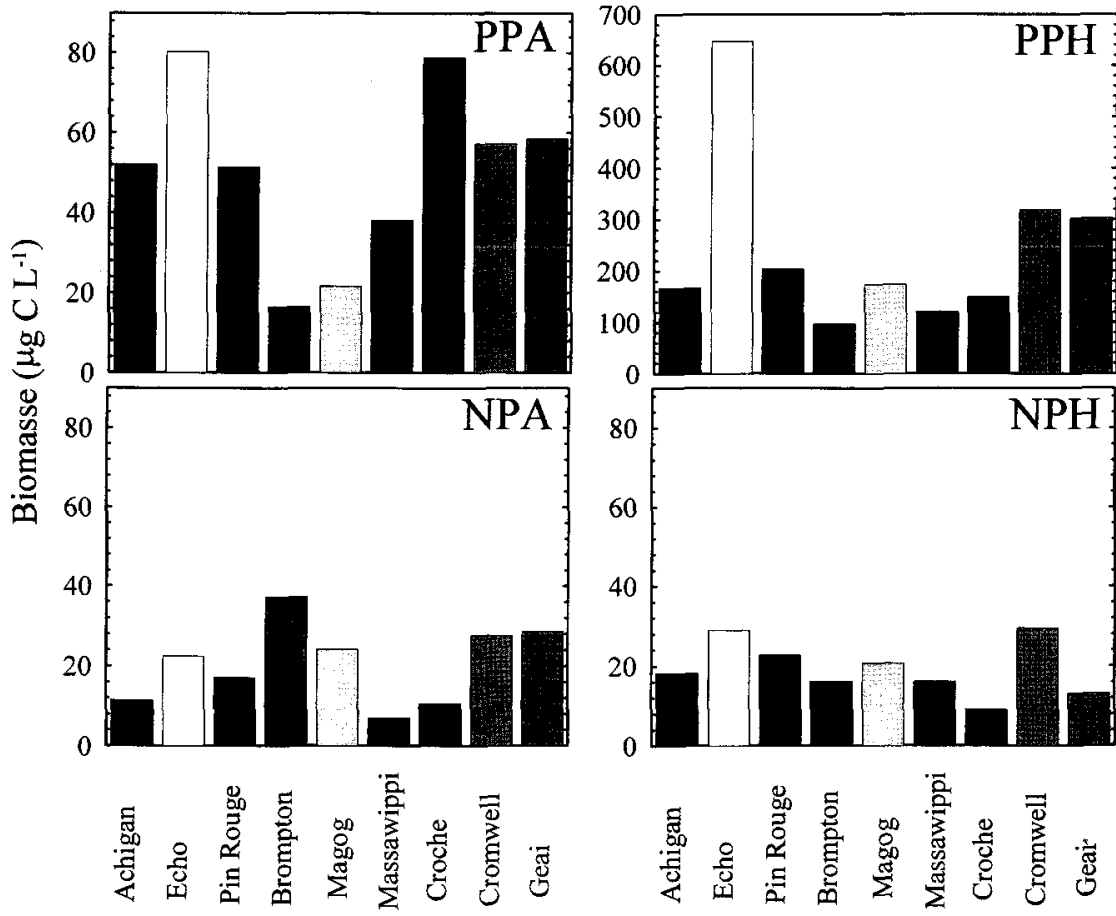

Figure 1 Biomasses moyennes $\left(\mu \mathrm{g} \cdot \mathrm{C} \cdot \mathrm{L}^{-1}\right)$ de picoplancton autotrophe (PPA) et hétérotrophe (PPH) et de nanoplancton autotrophe (NPA) et hétérotrophe (NPH) dans chacun des lacs des Laurentides et des Cantons de l'Est.

Mean biomass $\left(\mu \mathrm{g} \cdot \mathrm{C} \cdot \mathrm{L}^{-1}\right)$ of autotrophic (PPA) and heterotrophic $(P P H)$ picoplankton and of autotrophic (NPA) and heterotrophic (NPH) nanoplankton in each lake of the Laurentides and the Eastern Townships. 
En ce qui concerne les compartiments autotrophes, les biomasses de picoplancton autotrophe (PPA : 16,5 à $80 \mu \mathrm{g} \cdot \mathrm{C} \cdot \mathrm{L}^{-1}=75-365 \mathrm{mg} \cdot \mathrm{m}^{-3}$ ) observées dans les lacs du Québec sur un gradient en PT de 7 à $73 \mu \mathrm{g} \cdot \mathrm{L}^{-1}$ sont du même ordre de grandeur que celles rapportées par STOCKNER (1991) dans les lacs de la Colombie britannique ayant des statuts trophiques similaires (PT : 10$100 \mu \mathrm{g} \cdot \mathrm{L}^{-1}$; PPA : $\left.21-210 \mu \mathrm{g} \cdot \mathrm{C} \cdot \mathrm{L}^{-1}\right)$. Les biomasses moyenne de PPA dans les lacs des Laurentides sont 2 fois plus grandes $\left(61-65 \mu \mathrm{g} \cdot \mathrm{C} \cdot \mathrm{L}^{-1}\right)$ que celle notée dans les lacs des Cantons de l'Est $\left(25 \mu \mathrm{g} \cdot C \cdot \mathrm{L}^{-1}\right)$. Les biomasses de nanoplancton autotrophe (NPA) varient de 6,9 à $37,3 \mu \mathrm{g} \cdot \mathrm{C} \cdot \mathrm{L}^{-1}\left(46-249 \mathrm{mg} \cdot \mathrm{m}^{-3}\right)$ dans les lacs du Québec. Elles sont plus élevées que dans le lac Supérieur (MUNAWAR et al., 1978) mais similaires ou légèrement inférieures à celles des lacs Michigan et Huron (CARRICK et FAHNENSTIEL 1990) qui ont des niveaux de PT (5 et $8 \mu \mathrm{g} \cdot \mathrm{L}^{-1}$ ) similaires aux lacs oligotrophes des Laurentides. Les plus fortes biomasses de NPA sont notées dans les lacs Brompton et Magog des Cantons de l'Est $\left(37,3\right.$ et $\left.24,2 \mu \mathrm{g} \cdot \mathrm{C} \cdot \mathrm{L}^{-1}\right)$ et les lacs Cromwell, Geai et Écho des Laurentides $\left(27,6,28,6\right.$ et $\left.22,4 \mu \mathrm{g} \cdot \mathrm{C} \cdot \mathrm{L}^{-1}\right)$.

En ce qui a trait aux compartiments hétérotrophes, les biomasses de picoplancton hétérotrophe (PPH) dans les neufs lacs à l'étude varient entre 96,8 et $646,8 \mu \mathrm{g} \cdot \mathrm{C} \cdot \mathrm{L}^{-1}\left(440-2940 \mathrm{mg} \cdot \mathrm{m}^{-3}\right)$. Ces biomasses se comparent à celles observées dans les lacs d'Amérique du Nord (CURRIE, 1990) et du Québec (BIRD et KALFF, 1984). Les plus faibles biomasses de PPH se rencontrent dans les lacs des Cantons de l'Est $\left(129,8 \mu \mathrm{g} \cdot \mathrm{C} \cdot \mathrm{L}^{-1}\right.$ en moyenne). Le lac Écho se distingue par sa biomasse très élevée de $\mathrm{PPH}\left(645 \mu \mathrm{g} \cdot \mathrm{C} \cdot \mathrm{L}^{-1}\right)$ et deux autres lacs des Laurentides affichent aussi des biomasses importantes de PPH (Cromwell et Croche : 319 et $\left.304 \mu \mathrm{g} \cdot C \cdot L^{-1}\right)$. Les biomasses de nanoplancton hétérotrophe $(\mathrm{NPH})$ sont moins variables (9 à $29,5 \mu \mathrm{g} \cdot \mathrm{C}^{\mathrm{L}} \mathrm{L}^{-1}=60-196 \mathrm{mg} \cdot \mathrm{m}^{-3}$ ). Elles sont similaires à celles retrouvées dans les lacs Michigan et Huron $\left(2-24 \mu \mathrm{g} \cdot \mathrm{C} \cdot \mathrm{L}^{-1}\right)$ (CARRICK et FAHNENSTIEL 1989). Les plus fortes biomasses de NPH sont observées dans les lacs Écho et Cromwell $\left(28,9\right.$ et $\left.29,5 \mu \mathrm{g} \cdot \mathrm{C} \cdot \mathrm{L}^{-1}\right)$ et la plus faible au lac Croche $\left(9,1 \mu \mathrm{g} \cdot \mathrm{C} \cdot \mathrm{L}^{-1}\right)$ dans les Laurentides.

Dans l'ensemble des lacs, le picoplancton total (PPA + PPH) constitue une fraction très importante $(72-87 \%)$ de la biomasse totale des compartiments microbiens. Ceci est attribuable aux très fortes biomasses de PPH. Dans les lacs des Laurentides, la biomasse de PPA ne représente que 14 à $17 \%$ de la biomasse totale tandis que PPH compte pour 73 à $68 \%$. L'importance relative du nanoplancton dans les lacs des Laurentides est beaucoup plus faible (6 à $9 \%$ pour NPA et 7 à $8 \%$ pour NPH). Dans les lacs des Cantons de l'Est, la contribution du picoplancton autotrophe (PPA : $12 \%)$ et hétérotrophe (PPH : $60 \%$ ) à la biomasse totale est légèrement plus faible que celle notée dans les lacs des Laurentides. En revanche, les compartiments du nanoplancton y sont plus importants (NPA : $16 \%$ et NPH : $12 \%$ ).

\subsection{Variations inter-lacs du micro- et macrozooplancton}

La figure 2 présente la variation inter-lacs dans les densités du zooplancton total, des rotifères, des microcrustacés et des macrocrustacés. L'abondance du zooplancton et des guildes fonctionnelles varient beaucoup d'un lac à l'autre ; il n'y a pas de différences systématiques entre les sous-ensembles de lacs de Laurentides et des Cantons de l'Est. Ainsi, les plus fortes densités totales de zooplancton sont notées au lac Écho dans les Laurentides 

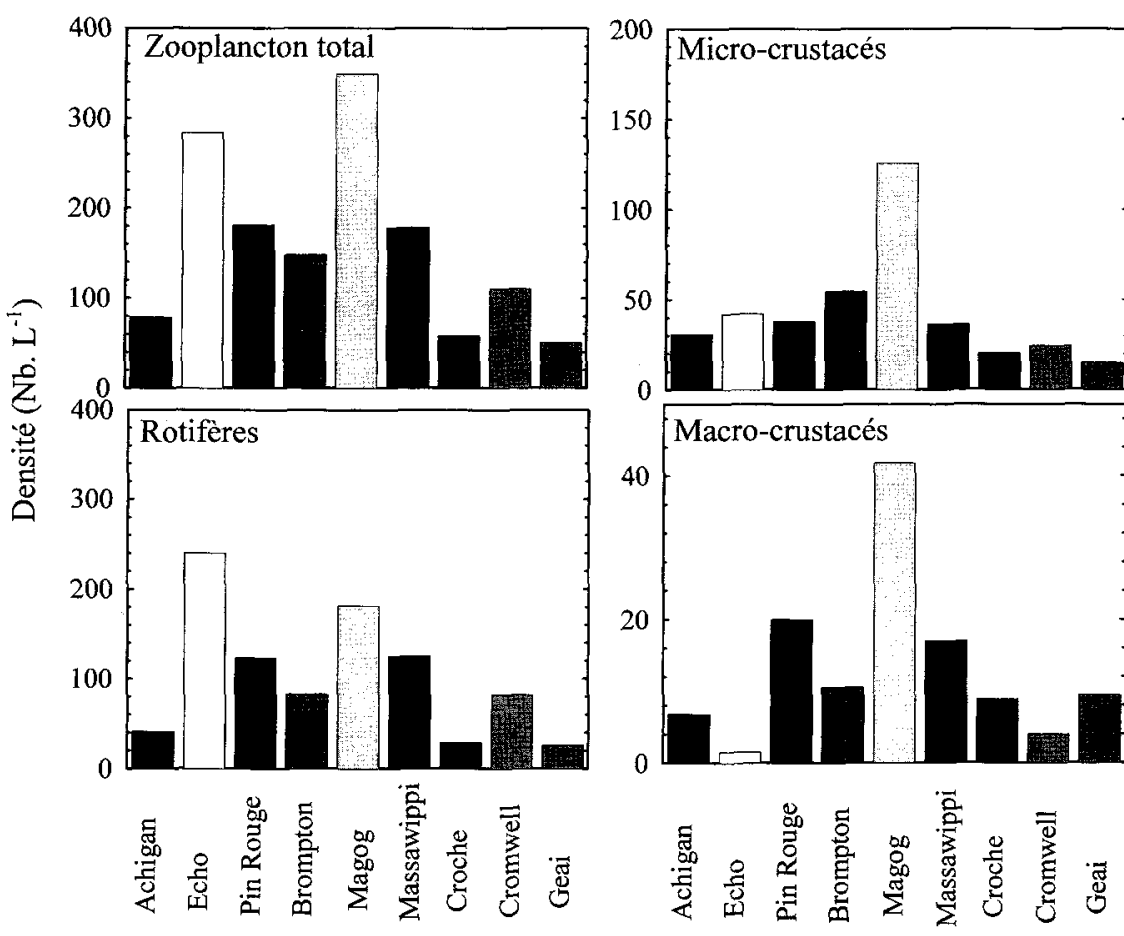

Figure 2 Densités moyennes (ind $\cdot \mathrm{L}^{-1}$ ) de zooplancton total, de rotifères, de microcrustacés et de macrocrustacés dans chacun des lacs des Laurentides et des Cantons de l'Est.

Mean densities (ind. $\mathrm{L}^{-1}$ ) of total zooplankton, rotifers, micro-crustaceans and macro-crustaceans in each lake of the Laurentides and the Eastern Townships.

$\left(283,7\right.$ ind $\left.\cdot \mathrm{L}^{-1}\right)$ et au lac Magog dans les Cantons de l'Est $\left(348,9\right.$ ind $\left.\cdot \mathrm{L}^{-1}\right)$. Trois lacs affichent des abondances moyennes (Pin Rouge, Brompton et Massawippi : 148-181 ind $\cdot L^{-1}$ ) et quatre lacs (Achigan, Croche, Cromwell et Geai) des abondances plus faibles $\left(50-110\right.$ ind $\left.\cdot L^{-1}\right)$. Les Rotifères étant prédominants en nombre ; on observe donc les mêmes patrons de variation interlacs pour la densité des rotifères et du zooplancton total. La densité des microcrustacés est très élevée au lac Magog (125,6 ind $\left.\cdot \mathrm{L}^{-1}\right)$. En moyenne, la densité des microcrustacés est plus forte dans les lacs des Cantons de l'Est $\left(72,2\right.$ ind $\left.\cdot L^{-1}\right)$ que dans les lacs des Laurentides $\left(19,9\right.$ et 37 ind $\left.\cdot L^{-1}\right)$. Les lacs des Cantons de l'Est sont également plus riches en macrocrustacés $\left(23,1\right.$ ind $\left.\cdot L^{-1}\right)$, en particulier le lac Magog, que les lacs des Laurentides $(7,4$ et 9,6 ind $\left.\cdot L^{-1}\right)$. Seul le lac Pin rouge est aussi riche en macrocrustacés que les lacs Brompton et Massawippi des Cantons de l'Est. Dans les lacs des Laurentides étudiés en 1992 (Croche, Cromwell et Geai), les densités de zooplancton total, de rotifères et de crustacés sont plus faibles (respectivement $72,6,45,3$, et $27,3 \mathrm{ind} \cdot \mathrm{L}^{-1}$ ) que dans les lacs des Laurentides étudiés en 1990 (en particulier Écho et Pin Rouge) (respectivement $181,4,134,8$ et 46,6 ind $\cdot L^{-1}$ ). En moyenne, les lacs des Cantons de l'Est supportent des densités de zooplancton total (225 ind $\left.\cdot \mathrm{L}^{-1}\right)$, de micro- $\left(72\right.$ ind $\left.\cdot \mathrm{L}^{-1}\right)$ et de macrocrustacés $\left(23\right.$ ind $\left.\cdot \mathrm{L}^{-1}\right)$ 
plus fortes que les sous-ensembles de lacs des Laurentides échantillonnés en 1990 (respectivement 181, 37 et 10 ind $\cdot \mathrm{L}^{-1}$ ) et en $1992\left(73,20\right.$ et 7,4 ind $\left.\cdot \mathrm{L}^{-1}\right)$. Pour les rotifères, les densités sont comparables dans les lacs des Cantons de l'Est et des Laurentides en 1990 (130 et 135 ind $\left.\cdot L^{-1}\right)$ mais plus élevés que dans les lacs des Laurentides en $1992\left(45,3\right.$ ind $\left.\cdot \mathrm{L}^{-1}\right)$. Le rapport microzooplancton/ macrozooplancton varie de minimum de 4,4 (lac Geai) ou 5,6 (lac Croche) à des maximum de 27 (Cromwell) et 188 (lac Écho). Il est en moyenne de 17,9 dans les lacs des Laurentides étudiés en 1990, surtout à cause du lac Écho, et de 8,7 et 8,8 dans les lacs des Cantons de l'Est et les lacs des Laurentides étudiés en 1992. Les variations du rapport micro-/macrozooplancton sont plus large d'un lac à l'autre qu'entre les trois sous-ensembles de lacs.

\subsection{Variations spatiotemporelles intra-lac des compartiments microbiens}

Les variations spatiales (entre les strates EPI, META, HYPO) et temporelles (entre les cinq dates d'échantillonnage) de la biomasse $\left(\mathrm{mg} \cdot \mathrm{m}^{-3}\right.$ ) des quatre compartements microbiens pour chacun des lacs sont illustrées à la figure 3. De façon générale, les résultats des tests ANOVA (tableau 2) démontrent qu'il existe une forte hétérogénéité inter-lacs (effet $L$ ) et des variations intra-lac de plus faible importance associées aux effets principaux $(p, d)$ et aux interactions simples $(L \times p, L \times d, p \times d)$ ou doubles $(L \times p \times d)$ entre les facteurs lac $(L)$, profondeur $(p)$ et date $(d)$.

La biomasse du picoplancton autotrophe (PPA) présente une forte variation inter-lacs au sein des trois sous-ensembles de lacs (tableau 2 ; figure $3 A$ ). Dans les lacs des Laurentides en 1990, le lac Écho a la plus forte biomasse de PPA tandis que les lacs Achigan et Pin Rouge ont des biomasses similaires et plus faibles. Parmi les lacs des Laurentides en 1990, le lac Écho supporte la plus grande biomasse de PPA. Dans les lacs des Cantons de l'Est, le lac Massawippi a la plus forte biomasse de PPA alors que les lacs Magog et Brompton présentent des biomasses 2 fois plus faibles. Dans les lacs des Laurentides en 1992, le lac Croche a la plus forte biomasse de PPA. II existe aussi des interactions hautement significatives entre les effets lac et profondeur $(L \times p)$ ainsi qu'entre les effets lac et date d'échantillonnage $(L \times d)$ (tableau 2) révélant des variations spatiotemporelles de PPA non constantes d'un lac à l'autre. Dans les lacs des Laurentides en 1990, la biomasse moyenne de PPA dans le lac Achigan est plus élevée dans l'épi- et le métalimnion que dans l'hypolimnion, tandis qu'elle ne varie pas selon la profondeur dans le lac Écho et qu'elle diminue avec la profondeur dans le lac Pin Rouge. De plus, les plus fortes biomasses s'observent à différentes dates d'échantillonnage dans chaque lac. Dans les lacs des Cantons de l'Est, la biomasse de PPA ne varie pas selon les strates dans les lacs Brompton et Magog alors qu'elle diminue dans I'hypolimnion au lac Massawipi. La biomasse de PPA dans les lacs des Cantons de l'Est varie également au cours de la saison estivale et elle atteint des maxima à différentes périodes dans chaque lac. Dans les lacs des Laurentides en 1992, la biomasse de PPA diminue dans l'hypolimnion au lac Croche tandis qu'elle ne varie pas selon les strates dans le lac Geai et qu'elle augmente dans l'hypolimnion au lac Cromwell. Au cours de la saison estivale, les lacs Cromwell et Geai présentent des biomasses maximales de PPA aux périodes 1, 2 et 4 tandis que dans le lac Croche, les plus fortes biomasses de PPA se rencontrent aux 

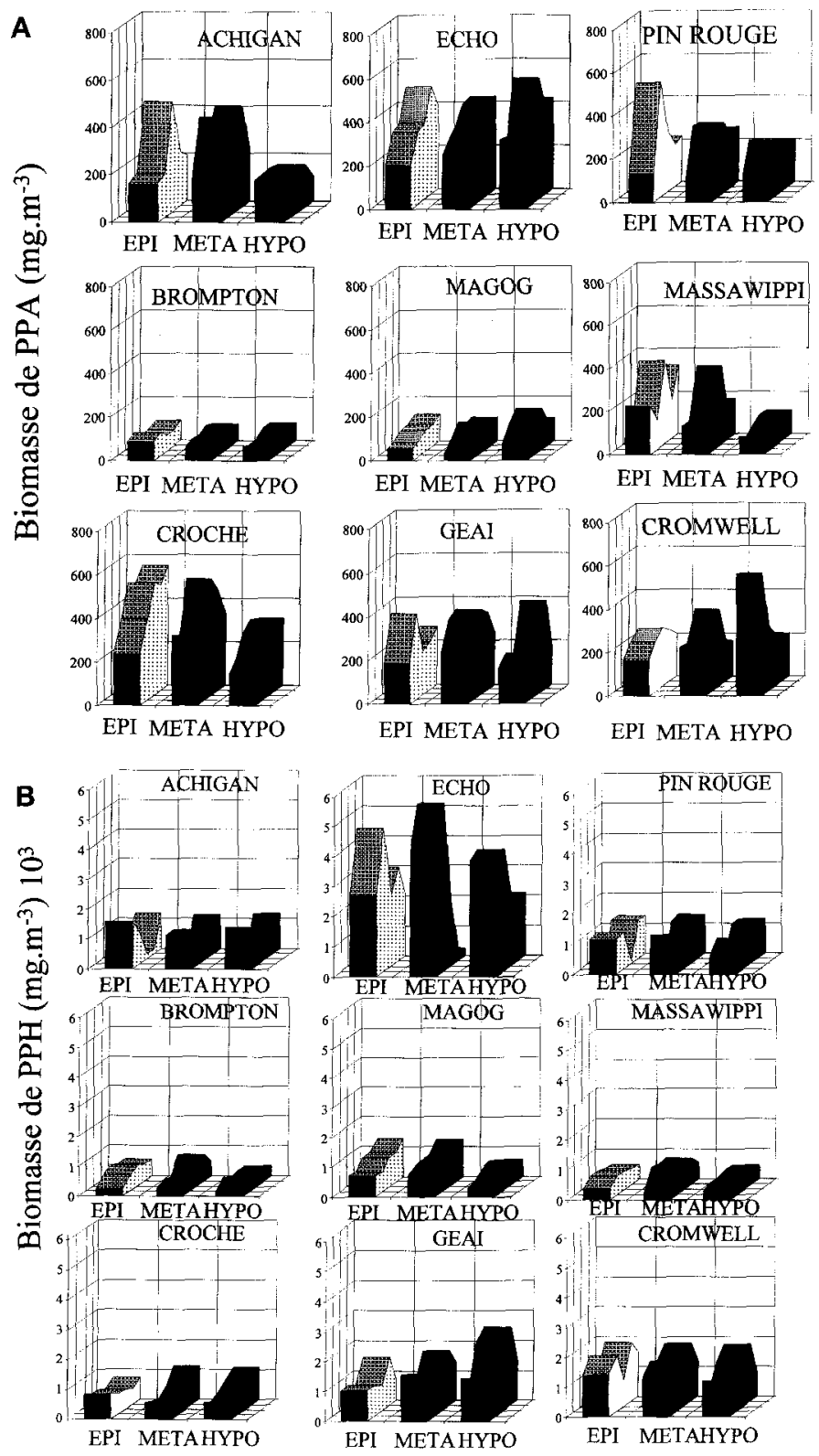

Figure 3 Variations spatiotemporelles des biomasses $\left(\mu \mathrm{g} \cdot \mathrm{C} \cdot \mathrm{L}^{-1}\right)$ de (A) picoplancton autotrophe (PPA), (B) de picoplancton hétérotrophe (PPH). La variation intra-lac dans l'espace et le temps est définie pour les strates limnétiques (EPI, META, HYPO) et les 5 dates d'échantillonnage.

Spatio-temporal variation of the biomass $\left(\mu \mathrm{g} \cdot \mathrm{C} \cdot \mathrm{L}^{-1}\right)$ of $(\boldsymbol{A})$ autotrophic picoplankton (PPA), (B) heterotrophic picoplankton (PPH). Within-lake varition in space and time are defined by the limnetic stratum (EPI, META, HYPO) and the 5 dates of sampling. 

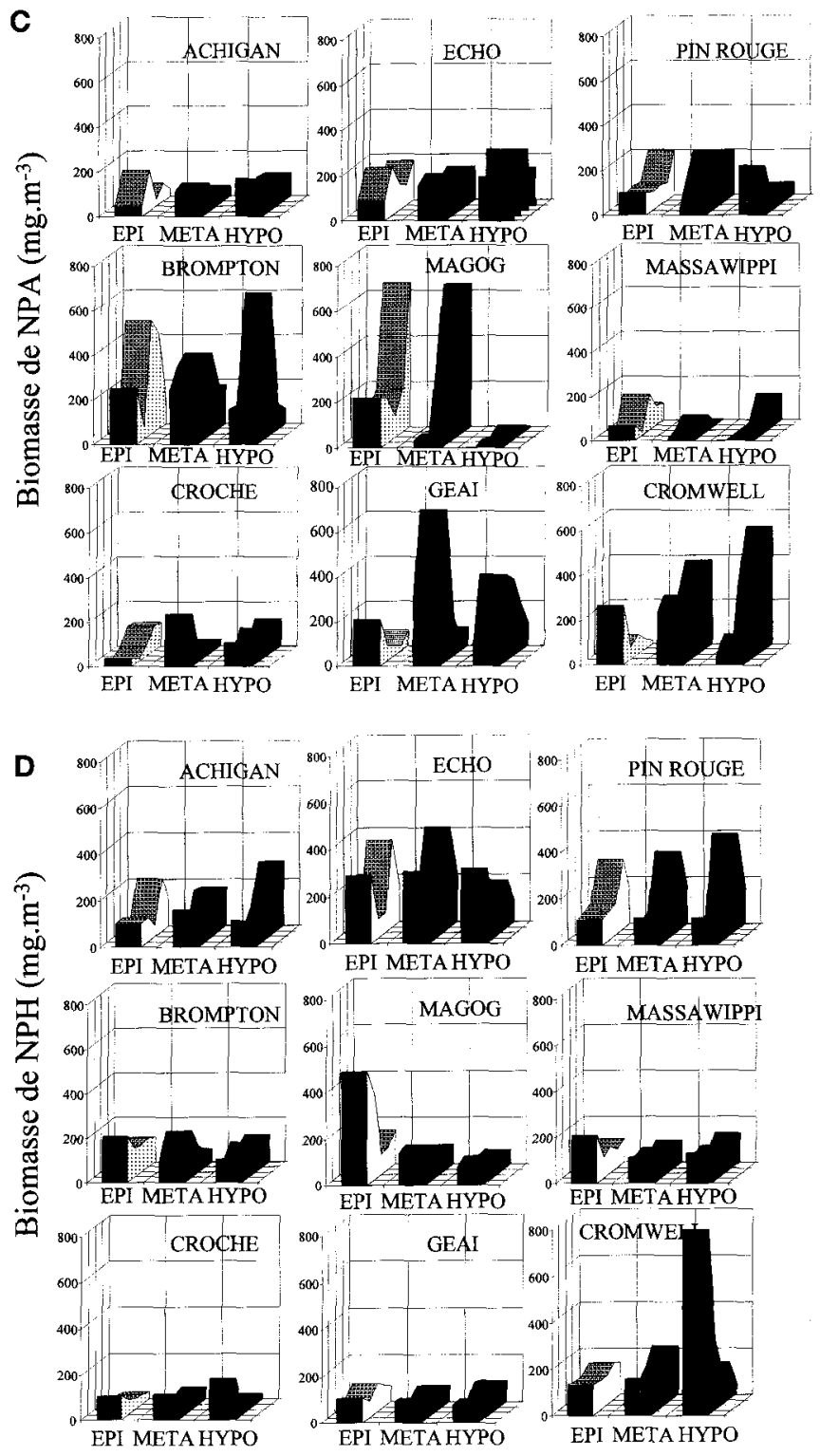

Figure 3 (suite) Variations spatiotemporelles des biomasses $\left(\mu \mathrm{g} \cdot \mathrm{C} \cdot \mathrm{L}^{-1}\right)$ de (C) nanoplancton autotrophe (NPA) et (D) nanoplancton hétérotrophe (NPH) dans chacun des lacs des Laurentides et des Cantons de l'Est. La variation intra-lac dans l'espace et le temps est définie pour les strates limnétiques (EPI, META, HYPO) et les 5 dates d'échantillonnage.

Spatio-temporal variation of the biomass $\left(\mu \mathrm{g} \cdot C \cdot \mathrm{L}^{-1}\right)$ of $(\mathrm{C})$ autotrophic nanoplankton (NPA), and (D) heterotrophic nanopalnkton in each lake of the Laurentides and the Eastern Townships. Within-lake varition in space and time are defined by the limnetic stratum (EPI, META, HYPO) and the 5 dates of sampling. 
Tableau 2 Résultats des ANOVAs testant les effets simples et des interactions des facteurs lac $(L)$, profondeur $(p)$ et date (d) sur les biomasses des quatre compartiments microbiens dans les trois sous-ensemble de lacs des Laurentides (1990 et 1992) et des Cantons de l'Est (1991).

Table 2 Results from ANOVAs testing principal effects and the interactions of factors lake (L), depth $(p)$ and date (d) on the biomass of the four microbial compartments in the three sets of lakes in the Laurentides (1990 and 1992) and the Eastern Townships (1991).

\begin{tabular}{|c|c|c|c|c|}
\hline $\begin{array}{l}\text { Compartiments } \\
\text { microbiens }\end{array}$ & $\begin{array}{l}\text { Effets prìncipaux } \\
\text { et interactions }\end{array}$ & $\begin{array}{c}\text { Laurentides } \\
1990\end{array}$ & $\begin{array}{c}\text { Cantons } \\
\text { de l'Est } \\
1991\end{array}$ & $\begin{array}{c}\text { Laurentides } \\
\quad 1992\end{array}$ \\
\hline \multirow{7}{*}{ PPA } & $L$ & $<0,0001$ & $<0,0001$ & $<0,0001$ \\
\hline & $P$ & ns & ns & ns \\
\hline & $d$ & ns & $\mathrm{ns}$ & ns \\
\hline & $L \times p$ & 0,0082 & $<0,0001$ & $<0,0001$ \\
\hline & $L \times d$ & 0,0051 & 0,0007 & $<0,0001$ \\
\hline & $p \times d$ & ns & ns & ns \\
\hline & $L \times p \times d$ & 0,0460 & ns & 0,0005 \\
\hline \multirow{7}{*}{ NPA } & $L$ & 0,0015 & $<0,0001$ & $<0,0001$ \\
\hline & $P$ & ns & ns & ns \\
\hline & $d$ & ns & $\mathrm{ns}$ & ns \\
\hline & $L \times p$ & ns & 0,0256 & 0,0058 \\
\hline & $L \times d$ & ns & 0,0207 & 0,0003 \\
\hline & $p \times d$ & ns & ns & ns \\
\hline & $L \times p \times d$ & ns & ns & 0.0039 \\
\hline \multirow{6}{*}{ PPH } & $\mathrm{L}$ & $<0,0001$ & $<0,0001$ & $<0,0001$ \\
\hline & $p$ & ns & 0,0452 & ns \\
\hline & $d$ & ns & 0,0248 & ns \\
\hline & $L \times p$ & $<0,0001$ & 0,0350 & $<0,0001$ \\
\hline & $L \times d$ & 0,0001 & 0,0281 & $<0,0001$ \\
\hline & $\begin{array}{c}p \times d \\
L \times p \times d\end{array}$ & $\begin{array}{c}\text { ns } \\
<0.0001\end{array}$ & $\begin{array}{c}\text { ns } \\
0,0536 \text { ns }\end{array}$ & $\begin{array}{c}n s \\
<0,0001\end{array}$ \\
\hline \multirow{7}{*}{ NPH } & L & 0,0281 & NS & $<0,0001$ \\
\hline & $p$ & ns & ns & ns \\
\hline & $d$ & 0,0103 & ns & ns \\
\hline & $L \times p$ & ns & 0,001 & ns \\
\hline & $L \times d$ & $\mathrm{~ns}$ & 0,0066 & 0,0005 \\
\hline & $p \times d$ & ns & ns & ns \\
\hline & $L \times p \times d$ & ns & ns & 0,0095 \\
\hline
\end{tabular}

périodes 3 et 4 . De plus, dans les lacs des Laurentides, on remarque qu'il existe une interaction double $(L \times p \times d)$ significative (tableau 2$)$ indiquant des variations de biomasse de PPA en profondeur et selon la date d'échantillonnage non systématiques d'un lac à l'autre.

Les variations de la biomasse de NPA sont de faible amplitude dans les lacs des Laurentides en 1990 où seul l'effet principal lac (L) est significatif (tableau 2 ; figure $3 B$ ). Le lac Écho a la plus forte biomasse de NPA tandis que le lac Achigan présente la plus faible biomasse, le lac Pin Rouge ayant une position intermédiaire. Dans les lacs des Cantons de l'Est, l'effet lac (L) de 
même que ses interactions simples sont tous significatifs (tableau 2). Le lac Massawippi montre la plus faible biomasse de NPA (figure 3B). De plus, la biomasse de NPA diminue fortement dans I'hypolimnion anoxique du lac Magog tandis qu'aucune variation entre les strates n'a été observée dans les lacs Brompton et Massawippi. De façon générale, la biomasse de NPA est plus importante à la fin de l'été dans les lacs des Cantons de l'Est. Dans les lacs des Laurentides en 1992, l'effet principal lac (L) de même que ses interactions simple et double avec les effets date $(L \times d)$ et profondeur $(L \times p)$ sont tous hautement significatifs (tableau 2). Le lac Croche a une biomasse de NPA au moins deux fois plus faible que celle observée dans les lacs Geai et Cromwell (figure $3 B$ ). De plus, la biomasse de NPA ne varie pas en fonction des strates au lac Croche tandis qu'elle est importante dans le métalimnion et I'hypolimnion aux lacs Cromwell et Geai. Les biomasses de NPA atteignent des maxima à différentes périodes dans chaque lac comme l'indique l'interaction triple $(\mathrm{L} \times$ $p \times d)($ tableau 2).

La biomasse du PPH montre une forte hétérogénéité inter-lacs au sein de chaque sous-ensemble de lacs (tableau 2 ; figure $3 \mathrm{C}$ ). Dans les lacs des Laurentides en 1990, le lac Écho a la plus forte biomasse de PPH tandis que les lacs Achigan et Pin Rouge ont des biomasses plus faibles (figure 3C). Dans les lacs des Cantons de l'Est, le lac Magog a la plus forte biomasse de PPH tandis que le lac Brompton a la plus faible biomasse (figure $3 \mathrm{C}$ ). Les interactions simples $(L \times p, L \times d)$ ou doubles $(L \times p \times d)$ entre les effets principaux sont significatives et indiquent que les variations spatiales et temporelles de la biomasse du PPH ne sont pas constantes d'un lac à l'autre (tableau 2). Dans les lacs des Cantons de l'Est, on remarque l'existence d'un effet principal profondeur et date significatif (tableau 2). Ce phénomène indique que les variations spatiales et temporelles de la biomasse de PPH dans les lacs des Cantons de l'Est suivent le même patron dans le temps (augmentation vers la fin de l'été), et dans l'espace (diminution dans l'hypolimnion) dans les trois lacs (figure $3 C$ ). Dans les lacs des Laurentides en 1992, les lacs Cromwell et Geai ont les plus fortes biomasses de PPH (figure 3C). Les interactions sont aussi significatives et reflètent une augmentation de la biomasse de PPH dans le métalimnion et l'hypolimnion des lacs Croche et Geai ainsi qu'une hausse des biomasses de $\mathrm{PPH}$ généralement en fin de saison. Toutefois, l'absence d'effets principaux $\mathrm{p}$ et $d$ indique que les variations spatiales et temporelles ne sont pas semblables dans tous les lacs (tableau 2).

La biomasse de NPH est la moins variable entre les lacs. La variation interlac $(L)$ est significative seulement dans les lacs des Laurentides (tableau 2) où certains lacs (Écho, Pin Rouge, Cromwell) ont des biomasse de NPH plus élevées (figure 3D). En revanche, dans les lacs des Cantons de l'Est, la faible différence de biomasses entre les lacs expliquent l'absence d'un effet lac (L) significatif. Les interactions double $(L \times p ; L \times d)$ sont significatives dans les lacs des Cantons de l'Est ainsi que les interactions $(L \times d)$ et $(L \times d \times p)$ dans les lacs des Laurentides en 1992 (tableau 2). Ces interactions indiquent que les variations spatiotemporelles de la biomasse du NPH ne sont pas constantes d'un lac à l'autre. Dans les lacs des Cantons de l'Est, la biomasse de NPH diminue dans l'hypolimnion anoxique du lac Magog tandis que dans les lacs Brompton et Massawippi, elle ne varie pas entre les strates. De plus, les plus fortes biomasses estivales s'observent à différentes périodes dans les trois lacs (figure $3 D$ ). L'existence de variations temporelles significatives (effet d) 
dans les lacs des Laurentides en 1990, indique que les biomasses de NPH suivent la même tendance temporelle dans tous les lacs avec des maxima saisonniers au début et à la fin de l'été (figure 3D). Dans les lacs des Laurentides en 1990 et en 1992, la biomasse du NPH ne varie pas selon les strates dans les trois lacs, comme l'indique l'absence d'un effet profondeur (p) et d'une interaction simple entre les facteurs lac et profondeur $(L \times p)$.

\subsection{Relations avec le statut trophique des lacs et la lumière}

Les biomasses de PPA et de PPH tendent à diminuer avec le statut trophique exprimé par le phosphore total (PT) (figure 4). Toutefois, il est difficile de définir une relation claire avec le niveau trophique et PT à cause du faible nombre de lacs avec des concentrations de PT $>20 \mu \mathrm{g} \cdot \mathrm{L}^{-1}$. Lorsque l'on considère la contribution relative des compartiments autotrophe et hétérotrophe à la biomasse microbienne $<20 \mu \mathrm{m}$, les tendances se maintiennent. En revanche, les biomasses de NPA et NPH ne présentent pas de tendance vraiment définie en fonction du gradient de phosphore (figure 4).
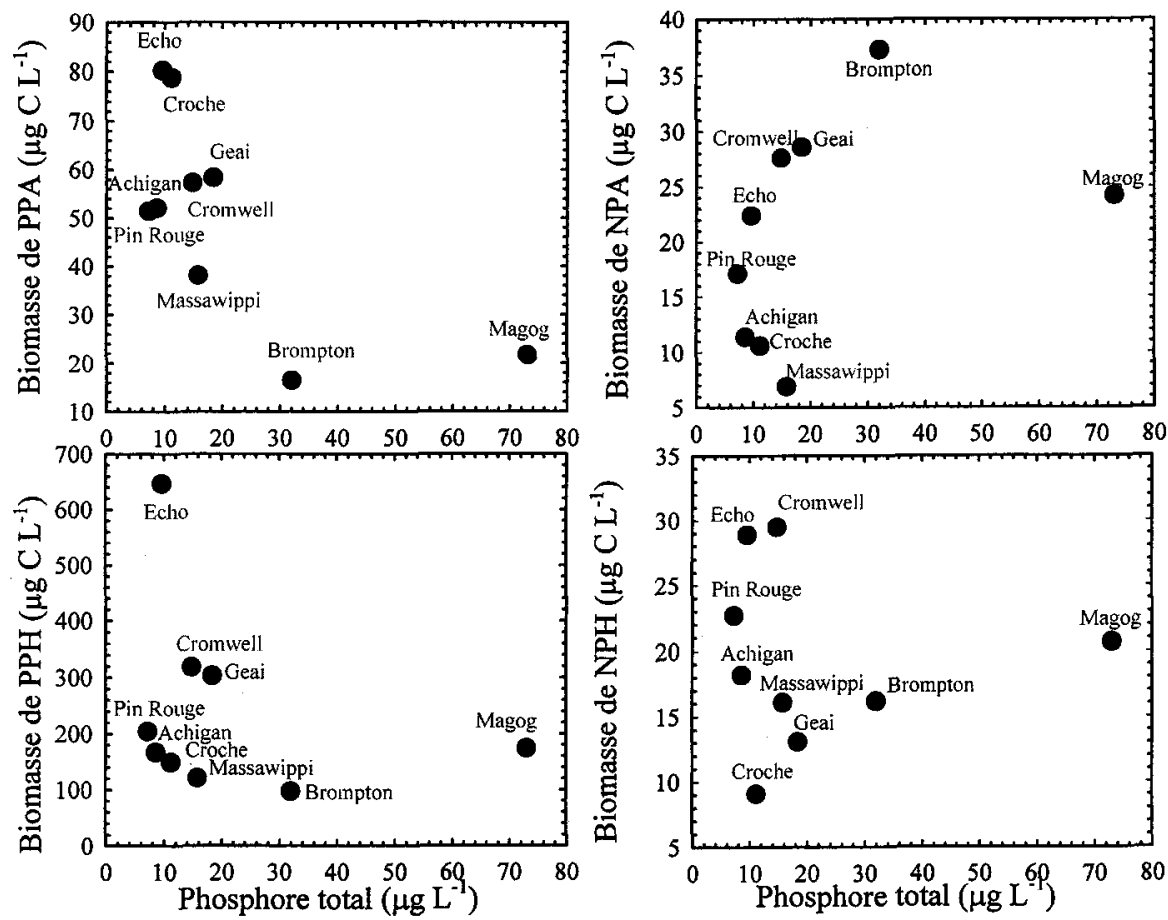

Figure 4 Relations entre les biomasses $\left(\mu \mathrm{g} \cdot \mathrm{C} \cdot \mathrm{L}^{-1}\right)$ de picoplancton autotrophe (PPA) et hétérotrophe (PPH), et de nanoplancton autotrophe (NPA) et hétérotrophe (NPH) avec le statut trophique des lacs, exprimé par la concentration en phosphore total (PT).

Relationships between the biomass $\left(\mu \mathrm{g} \cdot C \cdot \mathrm{L}^{-1}\right)$ of autotrophic picoplankton (PPA), heterotrophic picoplankton (PPH), autotrophic nanoplankton (NPA), and heterotrophic nanoplankton and lake trophicc status expressed by TP concentration. 
La biomasse de picoplancton autotrophe (PPA) est plus grande dans les milieux oligomésotrophes des Laurentides (figure 4). La dominance du PPA dans les écosystèmes oligomésotrophes est un phénomène bien connu qui s'explique principalement par la petite taille des cellules qui leur permet d'échapper aux herbivores zooplanctoniques ainsi que par leur rapport surface/volume élevé qui leur confère un avantage compétitif nutritionnel sur les cellules de plus grande taille dans les milieux pauvres en nutriments (FOGG, 1986 ; TEIXEIRA et GAETA, 1991 ; WEHR, 1991 ; PICK et AGBETI, 1991 ; PINELALLOUL et al., 1996). Cet avantage compétitif est perdu à mesure que les concentrations de nutriments augmentent (WEHR, 1990 ; LAFOND et al., 1990 ; TZARAs et al. 1999). Ainsi, dans les lacs eutrophes des Cantons de l'Est (Brompton, Magog), la biomasse de PPA est la plus faible tandis que la biomasse de NPA est plus forte (figure 4). Toutefois, dans le lac Massawippi peu productif et aux eaux dures et claíres, la biomasse de PPA est plus élevée que celle de NPA comme dans le cas des lacs oligomésotrophes des Laurentides. PICK (1991) a aussi démontré que les biomasses de PPA sont plus importantes dans les lacs aux eaux claires du Bouclier Canadien en Ontario.

La relation entre la biomasse de PPH et le statut trophique des lacs (PT) est moins claire que celle du PPA. En effet, la biomasse et l'importance relative du $\mathrm{PPH}$ tendent à diminuer selon le statut trophique mais la tendance est surtout due au lac Écho. On observe des biomasses de PPH du même ordre de grandeur (100-300 $\left.\mu \mathrm{g} \cdot \mathrm{C} \cdot \mathrm{L}^{-1}\right)$ dans les lacs ayant des concentrations de PT $<10 \mu \mathrm{g} \cdot \mathrm{L}^{-1}$ que dans ceux où $\mathrm{PT}$ est supérieure à $30 \mu \mathrm{g} \cdot \mathrm{L}^{-1}$ (figure 4 ). Pour le $\mathrm{NPH}$, il n'y a pas de tendance claire en fonction du statut trophique des lacs. TZARAS et al. (1999) ont évalué les effets d'addition de nutriments (phosphore, azote) sur les compartiments microbiens dans des enclos ; ils n'ont pas démontré de hausse de biomasse de NPH dans les enclos enrichis en nutriments.

\subsection{Relations avec le micro- et macrozooplancton}

Les variations de biomasse du picoplancton autotrophe et hétérotrophe entre les lacs peuvent être reliées aux changements dans la structure des communautés zooplanctoniques, en particulier l'importance relative du micro- et macrozooplancton. La biomasse de PPA est corrélée négativement à la densité de macrozooplancton $(r=-0,21 ; p=0,0001)$ et présente une tendance positive avec le rapport micro/macrozooplancton. En effet, les plus fortes biomasses de PPA se retrouvent dans les lacs des Laurentides (Écho, Croche, Cromwell, Geai) ayant les plus faibles densités de macrocrustacés (figures 2 et 5). Les lacs Écho et Cromwell sont également ceux qui ont les plus grand rapport micro-/macrozooplancton (188 et 27). Le développement de fortes biomasses de PPA dans les lacs où le rapport micro/macrozooplancton est élevé, est associé aux faibles densités de macrocrustacés, en particulier à l'absence de gros filtreurs comme les daphnies, qui peuvent exercer une très forte prédation sur le picoplancton autotrophe (WEISSE, 1988 ; STOCKNER, 1988 ; MAZUMDER et al., 1990).

Le picoplancton hétérotrophe (PPH) est aussi négativement corrélé à la densité du macrozooplancton $(r=-0,17 ; p=0,0001)$ et positivement au rapport micro-/macrozooplancton ( $r=0,08 ; p=0,02$ ). Les plus fortes biomasses de $\mathrm{PPH}$ sont observées dans les lacs Écho et Cromwell où les densités de 

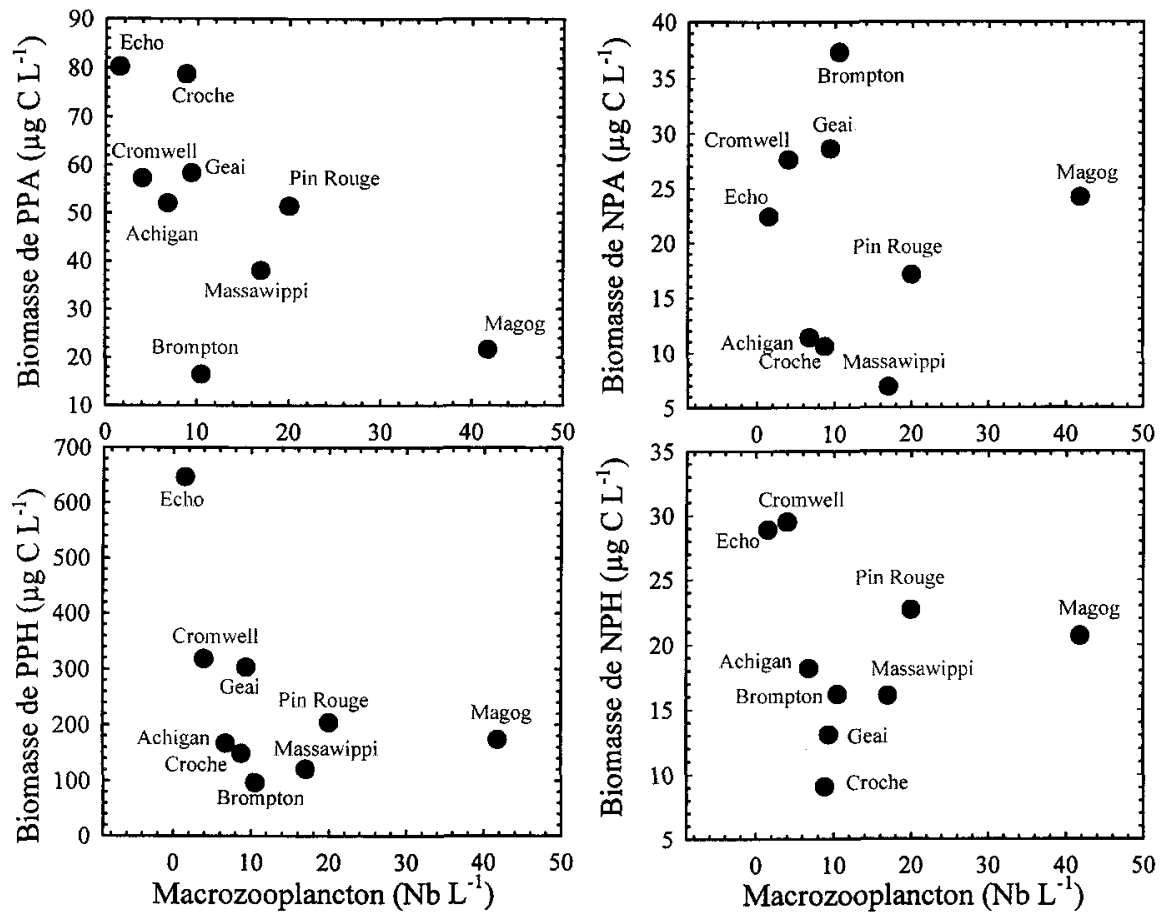

Figure 5 Relations entre les biomasses $\left(\mu \mathrm{g} \cdot \mathrm{C} \cdot \mathrm{L}^{-1}\right)$ de picoplancton autotrophe (PPA) et hétérotrophe (PPH), et de nanoplancton autotrophe (NPA) et hétérotrophe (NPH) avec la densité de macrozooplancton (ind $\cdot \mathrm{L}^{-1}$ ) dans les lacs à l'étude.

Relationships between the biomass $\left(\mu \mathrm{g} \cdot C \cdot \mathrm{L}^{-1}\right)$ of autotrophic picoplankton (PPA), heterotrophic picoplankton (PPH), autotrophic nanoplankton (NPA), and heterotrophic nanoplankton, and macrozooplankton density (ind. $L^{-1}$ ) in the study lakes.

macrozooplancton sont faibles $\left(<10 \mathrm{ind} \cdot \mathrm{L}^{-1}\right)$ et le rapport micro-/macrozooplancton élevé $(188,27)$ (figures 2 et 5). L'existence de fortes densités de macrocrustacés brouteurs est reconnue exercer une pression négative sur les bactéries (SIMON et TILZER, 1987 ; STOCKNER et SHORTREED, 1989 ; VAQUÉ et PACE, 1992). THOUVENOT et al., (1999b) ont montré que le macrozooplancton, en particulier Daphnia et Ceriodaphnia étaient responsables pour 56 à $75 \%$ des mortalités par broutage des bactéries dans un réservoir nouvellement mis en eau.

Les relations entre les biomasses de NPA et NPH et les densités de macrozooplancton ou le rapport micro-/macrozooplancton sont moins claires. Le groupe de lacs ayant les plus faibles densités de macrozooplancton (Cromwell et Écho) sont ceux qui supportent les plus fortes biomasse de NPA et NPH. Cependant, le lac Achigan, également pauvre en macrozooplancton, a des biomasses de NPA et NPH similaires ou plus faibles que les lacs Magog, Pin rouge et Massawippi, beaucoup plus riches en macrozooplancton. Dans le cadre d'expériences de biomanipulation en enclos, MAZUMDER et al. (1990) rap- 
porte que les fortes abondances de macrozooplancton dans les enclos sans poissons étaient associées à de plus faibles biomasses de NPA.

\section{4 - CONCLUSION GÉNÉRALE}

Cette étude est la première à décrire les variations spatiotemporelles des quatre composantes de la boucle microbienne dans une gamme de lacs représentatifs du sud du Québec. Bien que de nombreuses recherches aient été faites sur la boucle microbienne dans les milieux dulcicoles jusqu'à ce jour, trop peu d'entre elles ont analysé les quatre composantes de la boucle microbienne de façon simultanée ainsi que les facteurs environnementaux qui les influencent.

L'étude portait sur neuf lacs des régions des Laurentides et des Cantons de l'Est au sud du Québec dont le gradient trophique exprimé en PT, s'étendait de 7 à $73 \mu \mathrm{g} \cdot \mathrm{L}^{-1}$. La biomasse de picoplancton autotrophe (PPA) variait de 16 à $80 \mu \mathrm{g} \cdot \mathrm{C} \cdot \mathrm{L}^{-1}$ tandis que celle de picoplancton hétérotrophe (PPH) était 10 fois plus élevée et variait de 97 à $647 \mu \mathrm{g} \cdot \mathrm{C} \cdot \mathrm{L}^{-1}$. La biomasse de nanoplancton autotrophe variait de 7 à $37 \mu \mathrm{g} \cdot \mathrm{C} \cdot \mathrm{L}^{-1}$ tandis que celle de nanoplancton hétérotrophe variait de 9 à $29 \mu \mathrm{g} \cdot \mathrm{C} \cdot \mathrm{L}^{-1}$. Les biomasses des compartiments microbiens dans les lacs du Québec se comparent à celles observées ailleurs en Amérique du Nord, en particulier dans les lacs de la Colombie britannique, de l'Ontario et dans les Grands lacs.

La variation inter-lacs est la plus grande source de variabilité des biomasses des compartiments microbiens mais ils existent aussi des variations importantes intra-lac au niveau spatial entre les différentes strates limnétiques (épi-, méta-, hypolimnion) et au niveau temporel au cours de la saison estivale (juin-octobre). Les variations inter-lacs s'expliquent en fonction du statut trophique, exprimé par les concentrations de PT, et des densités de prédateurs métazoaires (macrozooplancton).

Les biomasses de picoplancton autotrophe (PPA) et hétérotrophe (PPH) tendent à diminuer dans les lacs plus eutrophes alors que la biomasse de NPA augmenterait. On observe les mêmes tendances lorsque l'on considère l'importance relative de ces compartiments à la biomasse planctonique $<20 \mu \mathrm{m}$. Le macrozooplanctonique exerce un effet de cascade trophique sur le picoplancton autotrophe (PPA) et hétérotrophe (PPH). En effet, les biomasses de picoplancton autotrophe (PPA) et hétérotrophe (PPH) sont plus importantes dans les milieux où les densités de macrozooplancton sont faibles (rapport microzoo/macrozooplancton élevé). Notre étude n'a pas démontré d'interactions de prédation du zooplancton sur les compartiments nanoplanctoniques, contrairement aux études expérimentales en enclos limniques. 


\section{REMERCIEMENTS}

Nous remercions P. Dutilleul de l'université McGill pour son apport indéniable lors des analyses statistiques. Nous voulons également souligner la collaboration de J. Désy, J. Gauthier, J. Gauvin, M. Trudel, L. Terreault et G. Méthot lors des échantillonnages et des analyses en laboratoire. Les analyses des nutriments ont été faites par M.-J. Carbonneau au laboratoire d'Y. Prairie de l'université du Québec à Montréal. J. Gauthier a effectué l'analyse du zooplancton en 1990 et 1992 et J. Gauvin l'analyse du zooplancton en 1991. Nos remerciements vont également au personnel de la Station de biologie de l'université de Montréal et à $\mathrm{C}$. Blanchard, secrétaire du GRIL, pour leur aide sur le terrain et la réalisation des figures. Cette étude a été rendue possible grâce à l'assistance financière du CRSNG et du Fonds FCAR.

\section{RÉFÉRENCES BIBLIOGRAPHIQUES}

AMBLARD C., BOURDIER G., SIMENGANDO T., RACHIQ S., CARRIAS J.-F., 1994. Diel and vertical variations of the microbial stocks (bacteria, heterotrophic flagellates, cilaites, phytoplankton) and their relative activities. Arch. Hydrobiol. Beih. Ergebn. Limnol., 41, 125-144.

AMBLARD C., BOURDIER G., CARRIAS J.F., MAURIN N., QUIBLIER C., 1996. Évolution saisonnière de la structure des communautés microbiennes dans un reservoir d'eau potable. Wat. Res., 30, 613-624.

BERGERON M., VINCENT W.F., 1997. Microbial food web responses to phosphorus supply and solar UV radiation in a subarctic lake. Aquat. Microb. Ecol., 12, 239-249.

BERTRAND N., VINCENT W.F., 1994. Structure and dynamics of photosynthetic picoplankton across the salt water transition zone of the St. Lawrence River. Can. J. Fish. Aquat. Sci., 51, 161-171.

BIRD D.F., KALFF J., 1984. Empirical relationships between bacterial abundance and chlorophyll concentration in fresh and marine waters. Can. J. Fish. Aquat. Sci., 41, 1015-1023.

BOOTH B.C., 1993. Estimating cell concentration et biomass of autotrophic plankton using microscopy. In: KEMP P.F, SHERR B.F., SHERR E.B., COLE J.J. (eds.), Handbook of Methods in Aquatic Microbial Ecology, Lewis Publishers, Boca Raton, Florida 33431, Chapter 24, 199-205.

BURNS C., SCHALLENBERG M., 1998. Impacts of nutrients and zooplankton on the microbial food web of an ultra-oligotrophic lake. J. Plankton Res., 20, 1501 1525.

CARON D.A. 1983. Technique for enumeration of heterotrophic and phototrophic nanoplankton, using epifluorescence microscopy and comparison with other procedures. Appl. Env. Microbiol., 46, 491-498.

CARON D.A., PICK F.R., LEAN D.R.S., 1985. Chroococcoid cyanobacteria in lake Ontario: vertical and seasonal distributions during 1982. J. Phycol., 21, 171175.

CARON D.A., GOLDMAN J.C., BENNETT M.R., 1988. Experimental demonstration of the roles of bacteria and bacterivorous protozoa in plankton nutrient cycles. Hydrobiologia, 159, 27-40.

CARRICK H.J., FAHNENSTIEL G.L., 1989. Biomass, size structure and composition of phototrophic and heterotrophic nanoflagellate communities in lakes Huron and Michigan. Can. J. Fish. Aquat. Sci., $46,1922-1928$. 
CARRICK H.J., FAHNENSTIEL G.L. 1990. Planktonic protozoa in lakes Huron and Michigan: seasonal abundance and composition of ciliates and dinoflagellates. J. Great Lakes Res., 16, 319-329.

CARRICK H.J., FAHNENSTIEL G.L., STOERMER E.F., WETZEL R.G., 1991. The importance of zooplancton-protozoan trophic couplings in lake Michigan. Limnol. Oceanogr., 36, 1335-1345.

CHRISTOFFERSEN K., RIEMANN B., KLYSNER A., SONDERGAARD M., 1993. Potential role of fish predation and natural populations of zooplankton in structuring a plankton community in eutrophic lake water. Limnol. Oceanogr., $38,561-573$.

CURRIE D.J., 1990. Large-scale variability and interactions among phytoplankton, bacterioplankton, and phosphorus. Limnol. Oceanogr., 35, 1437-1455.

DUTILLEUL P., 1993. Modifying the $t$-test for assessing the correlation between two spatial processes. Biometrics, 49, 305314.

DUTILLEUL P., PINEL-ALLOUL B., 1996. A doubly multivariate model for statistical analysis of spatio-temporal environmental data. Environmetrics, 7, 551-565.

FAHNENSTIEL G.L., CARRICK H.J., 1992. Phototrophic picoplankton in lakes Huron and Michigan: abundance, distribution, composition and contribution to biomass and production. Can. J. Fish. Aquat. Sci., 49, 379-388.

FOGG G.E., 1986. Picoplankton: Review lecture. Proc. R. Soc. Lond, B. 228, 1-30.

HOBBIE J.E., DALEY R.J., JASPER S., 1977. Use of nucleopore filters for counting bacteria by fluorescence microscopy. Appl. Environ. Microbiol., 33, 12251228.

JANSSON M., BERGSTRÖM A-K., BLOMQVIST P., ISAKSSON A., JONSSON A., 1999. Impact of allochtonous organic carbon on microbial food web carbon dynamics and structure in Lake Örträsket. Arch. Hydrobiol., 144, 409-428.

JÜRGENS K., 1994. Impact of Daphnia on planktonic microbial food webs - A review. Marine Microbial Food Webs, 8 , 295-324.

LAFOND M., PINEL-ALLOUL B., ROSS P.E., 1990. Biomass and photosynthesis of size-fractionated phytoplankton in Canadian Shield lakes. Hydrobiologia, 196, 25-38.

LEGENDRE P., VAUDOR A., 1991. Le progiciel $R$ : Analyse multidimensionnelle, analyse spatiale. Département de sciences biologiques, Université de Montréal, Montréal.

LETARTE Y., PINEL-ALLOUL B., 1991. Variations spatiotemporelles de l'abondance et de la production du bactérioplancton dans un lac humique du Bouclier canadien. Can. J. Microbiol., 37, 64-73.

LETARTE Y., HANSEN H.J., SONDERGAARD M., PINEL-ALLOUL B., 1992. Production and abundance of different bacterial-size classes : relationships with primary production and chlorophyll concentration. Arch. Hydrobiol., 126, 1526.

LORENSEN C.J., 1966. A method for the continuous measurement of in vivo chlorophyll concentration. Deep Sea Res., $13,223-227$

LUND J.W.G., KIPLING C., LE CREN E.D., 1958. The inverted microscope method of estimating algal numbers et the statistical basis of estimation by counting. Hydrobiologia, 11, 143-170.

MACISAAC E.A., STOCKNER J.G., 1993. Enumeration of phototrophic picoplankton by autofluorescence microscopy. In: KEMP P.F, SHERR B.F., SHERR E.B., COLE J.J (eds.), Handbook of Methods in Aquatic microbial Ecology. Lewis Publishers, Boca Raton, Florida 33431, Chapter 23, 187-197.

MALINSKY-RUSHANSKY N., BERMAN T., 1991. Picocyanobacteria and bacteria in lake Kinneret. Int. Revue ges. Hydrobiol., 76, 555-564.

MAZUMDER A., MCQUEEN D.J., TAYLOR W.D., LEAN D.R.S., DICKMAN M.D., 1990. Micro- and mesozooplankton grazing on natural pico- and nanoplankton in contrasting plankton communities produced by planktivore manipulation and fertilization. Arch. Hydrobiol., 118, 257-282.

MUNAWAR M., MUNAWAR I.F., CULP L.R., DUPUIS G., 1978. Relative importance of nannoplankton in lake Superior phytoplankton biomass and community metabolism. J. Great Lakes Res., 4, 462-480. 
NAGATA T., 1986. The seasonal abundance and vertical distribution of the $<3 \mu \mathrm{m}$ phytoplankton in the North bassin of Lake Biwa. Ecol. Res., 1, 207-221.

NAGATA T., 1988. The microflagellate-picoplankton food linkage in the water column of Lake Biwa. Limnol. Oceanogr., 33, 504-517.

NAKANO S., KOITABASHI T., UEDA T., 1998. Seasonal changes in abundance of heterotrophic nanoflagellates and their consumption of bacteria in Lake Biwa with special reference to trophic interactions with Daphnia galeata. Arch. Hydrobiol., 142, 21-34.

NÜRNBERG G.K., SHAW M., 1998. Productivity of clear and humic lakes: nutrients, phytoplankton, bacteria. Hydrobiologia, 382, 97-112.

PICK F.R., CARON D.A., 1987. Picoplankton and nanoplankton biomass in lake Ontario: relative contribution of phototrophic and heterotrophic communities. Can. J. Fish. Aquat. Sci., 44, 2164-2172.

PICK F.R., 1991. The abundance and composition of freshwater picocyanobacteria in relation to light penetration. Limnol. Oceanogr., 36, 1457-1462.

PICK F.R., AGBETI, M., 1991. The seasonal dynamics and composition of photosynthetic picoplankton communities in temperate lakes in Ontario, Canada. Int. Revue ges. Hydrobiol, 76, 565-580.

PINEL-ALLOUL B., MÉTHOT G., VERRAULT G., VIGNEAULT Y. 1990. Zooplankton species association in Quebec lakes: variation with abiotic factors, including natural and anthropogenic acidification. Can. J. Fish. Aquat. Sci., 47, 110-121.

PINEL-ALLOUL B., LETARTE Y., 1993. Relationships between small and large bacterioplankton and primary producers in Québec lakes. Verh. Internat. Verein. Limnol., 25.

PINEL-ALLOUL B., BOURBONNAIS N., DUTILLEUL P., 1996. Among-lake and within-lake variations of autotrophic pico- and naoplankton biomass in six Quebec lakes. Can. J. Fish. Aquat. Sci, 53, 2433-2445.

PORTER K.G., FEIG Y.S., 1980. The use of DAPI for identifying and counting aquatic microflora. Limnol. Oceanogr., 25 , 943-948.
PORTER K.G., 1988. Phagotrophic phytoflagellates in microbial food webs. Hydrobiologia, 159, 89-97.

RIEMANN B., SONDERGAARD M., 1986. Carbon dynamics in eutrophic temperate lakes. Elsevier Science Publishers. $284 \mathrm{p}$.

SANDERS R.W., PORTER K.G., BENNETT S.J., DEBIASE A.E., 1989. Seasonal patterns of bacterivory by flagellates, ciliates, rotifers and cladocerans in a freshwater planktonic community. Limnol. Oceanogr., 34, 673-687.

SIMON M., TILZER M.M., 1987. Bacterial response to seasonal changes in primary production and phytoplankton biomass in lake Constance. J. Plankton Res., 9, 535-552.

SOKAL R.R., ROHLF F.J., 1981. Biometry. The principles et practice of statistics in biological research. 2nd edition, Freeman. San Francisco, California, USA.

STOCKNER J.G., PORTER K.G., 1988. Microbial food web in freshwater planktonic ecosystems. In: CARPENTER S.R. (ed.), Complex interaction in lake communities. Springer-Verlag, New York, 71-84.

STOCKNER J.G., SHORTREED K.S., 1989. Algal picoplankton production and contribution to food-webs in oligotrophic British Columbia lakes. Hydrobiologia, 173, 151-166.

STOCKNER J.G., 1988. Phototrophic picoplankton: an overview from marine and freshwater ecosystems. Limnol. Oceanogr., 33, 765-775.

STOCKNER J.G., 1991. Autotrophic picoplankton in freshwater ecosystems: The view from the summit. Int. Revue ges. Hydrobiol., 76, 483-492.

STRICKLAND J.D.H., PARSONS T.R., 1972. A practical handbook of seawater analysis. Bull. Fish. Res. Board Can., 67, $311 \mathrm{p}$.

TEIXEIRA C., GAETA S.A., 1991. Contribution of picoplankton to primary production in Estuarine coastal and equatorial shallow lakes. Hydrobiologia, 209, 117122.

THOUVENOT A., RICHARDOT M., DEBROAS D., DEVAUX J., 1999a. Impact of natural metazooplankton assembalge on planktonic microbial 
communities in a newly flooded reservoir. J. Plankton Res., 21, 179-199.

THOUVENOT A., RICHARDOT M., DEBROAS D., DEVAUX J., 1999b. Bacterivory of metazooplankton, cillaites and flagellates in a newly flooded reservoir. J. Plankton Res., 21, 1659-1679.

TRANVIK L.J., 1992. Allochtonous dissolved organic matter as an energy source for pelagic bacteria and the concept of the microbial loop. Hydrobiologia., 229, 107114.

VAQUÉ D., PACE M.L., 1992. Grazing on bacteria by flagellates and cladocerans in lakes of contrasting food-web structure. J. Plankton Res., 14, 307-321.

VOLLENWEIDER R.A., TALLING J.T., WESTLAKE D.F., 1974. A manual on methods for primary production in aquatic environments. 2nd ed., Blackwell Scientific Publications, Oxford. 228p.

WEHR J.D., 1991. Nutrient and grazermediated effects on picoplankton and size structure in phytoplankton commu- nities. Int. Revue ges. Hydrobiol., 76, 643-656.

WEHR J.D., 1993. Effects of experimental manipulations of light et phosphorus supply on competition among picoplankton et nanoplankton in an oligotrophic lake. Can. J. Fish. Aquat. Sci., 50, 936-945.

WEISSE T., 1988. Dynamics of autotrophic picoplankton in lake Constance. J. Plankton Res., 10, 1179-1188.

WEISSE T., 1990. Trophic interactions among heterotrophic microplankton, nanoplankton and bacteria in lake Constance. Hydrobiologia, 191, 111-122.

WEISSE T., 1991. The microbial food web and its sensitivity to eutrophication and contaminant enrichment: a cross-system overview. Int. Revue ges. Hydrobiol., 76, 327-337.

ZHANG Y., PREPAS E.E., 1996. Short-term effects of $\mathrm{Ca}(\mathrm{OH})_{2}$ additions on phytoplankton biomass: a comparison of laboratory and in situ experiments. Water Research, 30, 1285-1294. 